\title{
Der vermeintliche Siegeszug der Digitalisierung
}

„Der Siegeszug der Digitalisierung ist nicht aufzuhalten.“ So oder so ähnlich liest man es derzeit häufig in Einleitungen von studentischen Hausarbeiten, hört es in den Medien (Breitkopf, 2015) oder aus der Industrie (Siemens, 2017) und in etlichen Büchern, die sich dem vermeintlichen Siegeszug in Vergangenheit, Gegenwart und Zukunft widmen (Barrat, 2013; Kurzweil, 2005; Nassehi, 2019; Rid, 2017; Tegmark, 2018). Selbstredend kommen alle, die den Satz auf seinen Wahrheitsgehalt prüfen, schnell zu dem Ergebnis, dass er in dieser Bestimmtheit ohne gewisse Einschränkungen oder Relativierungen gar nicht geäußert werden könne. So lassen sich auf dem Zug der Digitalisierung von Sieg zu Sieg etliche Irrwege und vermeintliche Niederlagen aufzeigen. Hiervon ganz abgesehen, ist die Digitalisierung anders als plakativ unterstellt jedoch zunächst einmal kein eigenständig handelnder Sieger. Das Postulat einer unentwegt von Sieg zu Sieg eilenden und dergestalt anthropomorphisierten Digitalisierung lässt folglich zwei unabdingbare Bezugsgrößen völlig außen vor: den Menschen und seine Gesellschaft. Ohne den Menschen ist Digitalisierung eine leere Hülse, ja zunächst einmal überhaupt nicht denkbar. Ohne Gesellschaft und die in ihr praktizierte Arbeitsteilung könnte die Welt nur schwerlich in einem solchen Umfang digitalisiert werden, wie der Mensch dies Stand heute beobachten kann. Digitalisierung wird primär für und durch die Gesellschaft vorangetrieben (Wittpahl, 2017). Dreh- und Angelpunkt von Digitalisierung ist mithin das Individuum, eingebettet in eine soziale Situation innerhalb der Gesellschaft. Nur vor diesem Hintergrund lassen sich Erfolge und Misserfolge der Digitalisierung also überhaupt beurteilen. Und nur mit Blick auf in die Zukunft gerichtete individuelle und gesellschaftliche Erwartungen und Prognosen sowie deren (Nicht-)Eintreffen ist der Siegeszug folglich zu begutachten.

Doch was meint nun überhaupt Digitalisierung in ihrem untrennbaren Verhältnis zu Mensch und Gesellschaft und wieso sollte eine dahingehend selbstreflexive 
Analyse der an sie gerichteten Erwartungen von Bedeutung sein? Dem Grundverständnis nach ist die Digitalisierung vordergründig nichts weiter als ein technischer Prozess, bei dem analoge Entitäten in ein digitales, meist binäres Format übertragen werden, mit dem Ziel, dieses mit Hilfe informationstechnischer Infrastrukturen wie bspw. dem Computer zu speichern und zu verarbeiten (Brennen \& Kreiss, 2016; Chun \& Soderman, 2008; Storsul \& Fagerjord, 2008). Schon hier zeigt sich, dass Digitalisierung im ursprünglichen Sinne lediglich auf binär codierte Information abstellt, ein dann digitales Datum. Jedoch verbirgt sich hinter jenem Phänomen, das wir insbesondere in Deutschland mit jenem Oberbegriff Digitalisierung bezeichnen, eine Vielzahl an unterschiedlichen Begriffen, Phänomenen, Erzählungen und Vorstellungen. ${ }^{1}$ Die Erzählungen beginnen hierbei meist mit einer einfachen Betrachtung von elektronischen Artefakten wie dem Computer im Zusammenspiel mit seinem menschlichen Nutzer und enden nicht selten in einer durch eben jene elektronischen Datenverarbeitungsanlagen ausgelösten Transformation menschlicher Gesellschaften oder gar gleich in einer vollständigen digitalen Transzendenz menschlicher Biologie und Erfahrung etwa, wenn es um Vorstellungen davon geht, die menschliche Seele in einen Computer ,hochzuladen“ (Barrat, 2013; Bostrom, 2016; Callaghan et al., 2017; Kurzweil, 2005; Tegmark, 2018). Etwas nüchterner betrachtet begründet sich der

\footnotetext{
${ }^{1}$ Es sei an dieser Stelle darauf verwiesen, dass der Begriff Digitalisierung im Deutschen umfassender gebraucht wird als im Englischen. Wenn dort vom technischen oder sozialen Phänomen Digitalisierung die Rede ist, wird meist entsprechend genauer differenziert: So meint Digitization meist nur die Abbildung von Informationen in einem digitalen Format (Storsul \& Fagerjord, 2008), wenn es um soziale Konsequenzen geht, wird häufig von Computerization, , digital Revolution“ etc. gesprochen (Kling \& Iacono, 1988). Zwar definieren auch Brennen und Kreiss (2016): "digitalization as the way many domains of social life are restructured around digital communication and media infrastructures" (S. 1). Eine einfache Übersetzung in Digitalization ist jedoch weithin weniger gebräuchlich und auch nicht zielführend, genauso wie die undifferenzierte Verwendung des Digitalisierungslabels zugunsten einer konkreten Benennung dessen, worüber man gerade spricht, vermieden werden sollte (Mahn, 2019). In loser Assoziation subsumieren sich in der deutschsprachigen Verwendung unter diesem Oberbegriff die materiellen Dinge der Digitalisierung, also die technischen Artefakte wie bspw. Computer oder Smartphone sowie auch die Infrastruktur des Internets mit ihren Rechenzentren, Glasfaserkabeln und Satelliten. Über diese greifbaren Objekte betrifft der Begriff Digitalisierung darüber hinaus jedoch auch weitreichendere Konzepte wie die sogenannten Sozialen Medien, vage Konzepte wie Industrie 4.0, das Internet of Things (IOT) usw. In diesen Konzepten kommt der Digitalisierung dann noch konkreter eine sozio-technologische Bedeutung zu, nämlich beim Einsatz von Computertechnologie als ,Werkzeug ' durch den Menschen. Der Mensch prägt ihre Verwendung und ihre Verwendung prägt den Menschen, ohne hier einen Technikdeterminismus zu unterstellen (Smith \& Marx, 1994; Wyatt, 2008).
} 
vermeintliche Siegeszug auf der vielfältigen, beinahe alle menschlichen Lebensbereiche durchdringenden Verwendung von Computern und den vordergründig positiven Veränderungen, die diese Entwicklung mit sich bringt. Der vermeintliche Gewinn besteht für das menschliche Individuum und seine Gesellschaft darin, einen Nutzen aus dieser fortschreitenden Digitalisierung zu ziehen, also Vorteile mit Blick auf Wirtschaft, Politik, Gesundheit, Kommunikation etc. zu erfahren, die ohne Digitalisierung so nicht möglich sind. Dabei kommt es mitunter auch zu überzogenen Zukunftserwartungen, was Digitalisierung leisten kann und soll. So formuliert der Journalist Jürgen Kaube mit Blick auf die Digitalisierung der Schule folgende Beobachtung (Kaube, 2019): „Man muss heute in jedem Lebensbereich nur noch ,Digitalisierung' sagen, und schon meldet sich der Verstand zugunsten von Visionen $a b^{\prime \prime}(\S 3)$. Was ist dran an dieser Aussage? Dient der Begriff Digitalisierung womöglich als eine ,Applaus!'-Anzeige, bei deren Aufleuchten das Publikum angehalten ist unreflektiert und ohne tatsächlichen Anlass mitzuklatschen (Yudkowsky, 2018)? Ist der Begriff bloß ein Feigenblatt, das vor einer tiefergehenden Betrachtung und kritischen Nachfragen schützt, was und warum da nun wie durch Hard- und Software ergänzt, verändert, oder gar verbessert werden soll? Glauben Bürger*innen in Deutschland vielleicht tatsächlich, dass jede Maßnahme, die nur das Label Digitalisierung trägt, zum Selbstläufer wird, bei dem sich der Erfolg von selbst einstellt? Der gesellschaftliche Umgang mit der Digitalisierung erscheint vielschichtiger und uneindeutiger, als es das digitale Kodierschema von 1 und 0 für Erfolg oder Misserfolg der Digitalisierung suggeriert.

Schnell stehen wirkmächtige Begriffe im Raum wie bspw. die digitale Revolution, von der erwartet wird, dass sie nach und nach alle Gesellschaftsbereiche erfasst, durchdringt und nachhaltig verändert (Brown, 2019; Helbing, 2019; Isaacson, 2014; Kelly, 2009; Schirrmacher, 2015; Zysman \& Newman, 2006). Dass Revolutionen - und nun eben auch die digitale - für gewöhnlich nicht zwangsläufig zum Vorteil aller Beteiligten verlaufen und naturgemäß auch mit sozialen Kosten und Nachteilen verbunden sind, darf mit Blick auf den durch Digitalisierung ausgelösten sozialen Wandel, der den Menschen direkt und unmittelbar betrifft, an dieser Stelle nicht vernachlässigt werden. Ein Beispiel: Nach anfänglicher Euphorie über die vermeintlich demokratisierende und befreiende Kraft des Internets (Ghonim, 2012; Shirky, 2008) - einer dezentralen Infrastruktur der Digitalisierung mit zeitgleich zentraler gesellschaftlicher Bedeutung - melden sich auch immer wieder Stimmen, die hieran berechtigte Zweifel anmeldeten, wenn bspw. auf den gesellschaftlich nachteiligen Missbrauch der Technik durch autokratische Herrschaft oder Unternehmen verwiesen wird (Eubanks, 2018; Morozov, 2012, 2014; Mosco, 2014; Pariser, 2011; Sunstein, 2002). 
Unter der Annahme, Digitalisierung sei für Individuum und Gesellschaft zumindest mit weitreichenden Konsequenzen behaftet und bedeutungsvoll, gebieten die diesbezüglich ablaufenden gesellschaftlichen Kommunikations- und Aushandlungsprozesse eine sozialwissenschaftliche Analyse. Die zuvor angesprochenen latenten, also nicht-beobachtbaren sozialen Konsequenzen der Digitalisierung können sozialwissenschaftlich analysiert werden, um sie trotz oder genau wegen ihrer Nicht-Einsehbarkeit sichtbar und verständlich zu machen.

Dabei scheinen insbesondere die an die Konsequenzen der Digitalisierung geknüpften Vorstellungen und Erwartungen für das soziale Miteinander in menschlichen Gesellschaften ein lohnenswertes Unterfangen auf dem Weg, die forschungsleitende Frage zu beantworten: Was und wie denken Menschen über die Digitalisierung und wozu führt dieses Denken? Es gibt hier viele spezifische Erzählungen zur Digitalisierung, die ihre gesellschaftliche Wahrnehmung prägen und die spezifische Vorstellungen auszulösen vermögen, was Digitalisierung für den Menschen und das menschliche Miteinander im Rahmen sozialer Ordnung bedeutet. Bezugnehmend auf den insbesondere im anglophonen Raum eng mit der Digitalisierung verbundenen Begriff ,Cyber" adressiert Rid (2017) dessen vielschichtige gesellschaftliche Bedeutung folgendermaßen:

For politicians in Washington, the word stands for power outages that could plunge entire cities into chaos at any moment. For spies in Maryland, it stands for conflict and war, and for data being stolen by Russian criminals and Chinese spies. For executives in the City of London, it stands for major security breaches, for banks bleeding money, and for ruined corporate reputations. For inventors in Tel Aviv, it triggers visions of humans merging with machines, of wired-up prostheses with sensitive fingertips, and of silicon chips implanted under tender human skin. For science fiction fans in Tokyo, it stands for an escapist yet retro punk aesthetic, for mirrored shades, leather jackets, and worn-down, dusty gadgets. For romantic internet activists in Boston, it stands for a new realm of freedom, a space beyond the control of oppressive governments and law enforcement agencies. For engineers in Munich, it stands for steely control, and for running chemical plants by computer console. Ageing hippies in San Francisco nostalgically think back to wholeness and psychedelics and 'turning on' the brain. And for screen-addicted youth in between, 'cyber' means simply sex-by-video-chat. (S. XIII)

Die Vielschichtigkeit der Digitalisierung als soziales Phänomen bedeutet letztlich auch, dass es nicht die sozialwissenschaftliche Digitalisierungsforschung geben kann, sondern sich einzelne wissenschaftliche Disziplinen mit der sozialen Dimension bestimmter Phänomene und Kontexte der Digitalisierung befassen. Es ist daher notwendig, zunächst ein paar einführende Worte zur Einordnung der vorliegenden Arbeit in den sozialwissenschaftlichen Forschungskontext zur 
Digitalisierung zu verlieren, um deutlich $\mathrm{zu}$ machen, auf welche Fragen die Leser*innen Antworten erwarten können und auf welche nicht.

\subsection{Erkenntnisinteressen und Forschungsobjekte der Digitalisierungsforschung}

Sozialwissenschaftliche Digitalisierungsforschung lässt sich nur schwer umfassend beschreiben und klar von anderen, nicht digitalisierungsbezogenen Forschungsbemühungen abgrenzen. Hierfür gibt es diverse Gründe: Zum einen ist das verbindende Element aller Digitalisierungsforschung lediglich das Materialobjekt, also die materiellen Artefakte der Digitalisierung selbst, auch wenn diese das zu untersuchende Phänomen nur mittelbar ermöglichen. Hier seien vor allem digitale ,Dinge“ wie zuallererst der Computer oder verwandte technologische Innovationen wie bspw. Smartphones genannt (Dogruel, 2013; Neuberger, 2008; Wagner, 1997). Das, was in engerem Sinne durch diese Computer-Artefakte beeinflusst und folglich untersucht wird, unterscheidet sich dann je nach dem sogenannten Formalobjekt der unterschiedlichen wissenschaftlichen Disziplinen.

Hierzu gehört dann bspw. die Forschung im Bereich Social Robotics, mit einem eher materiellen Fokus auf technische digitalisierte Artefakte an der Schnittstelle der Human-Computer-Interaction (Goodrich \& Schultz, 2007). So findet sich Forschung, die die menschliche Wahrnehmung in der Interaktion mit computerisierten Maschinen wie Pflegerobotern untersucht und bspw. Fragen nach deren Akzeptanz stellt (Heerink et al., 2010; Hudson et al., 2017), neben Untersuchungen zu den durch Digitalisierung und Automatisierung ausgelösten Veränderungen auf dem Arbeitsmarkt (Savela et al., 2017), die zeigen, dass Menschen durchaus mit Emotionen wie etwa durch Bedrohungswahrnehmungen ausgelöste Angst auf vermeintlich autonome Maschinen reagieren (Liang \& Lee, 2017).

Wenn man einmal auf die Kommunikationswissenschaft abstellt, ist das Formalobjekt die ,(soziale) Kommunikation“ (K. Beck, 2017), die mithin auch im Blickpunkt der vorliegenden Arbeit steht. Es interessiert also nicht die Digitalisierung an sich, sondern von Interesse sind die Bedingungen und Konsequenzen ihrer kommunikativen Verhandlung innerhalb der Gesellschaft. Doch auch die Betrachtung der Digitalisierung im Rahmen dieser sozialen Kommunikation ist vielfältig. So gibt es kommunikationswissenschaftliche Forschung, die einerseits die Bedingungen und Auswirkungen computervermittelter interpersonaler Kommunikation betrifft und andererseits digitalisierte öffentliche massenmediale Kommunikation in den Blick nimmt (K. Beck, 2006; Kimpeler et al., 2007; 
Schweiger \& Beck, 2010). Zu nennen sind hier allen voran die sogenannten ,Sozialen“ Medien mit einem Fokus auf Kommunikation in den Onlineangeboten sozialer Netzwerke (Hautzer et al., 2012; J.-H. Schmidt, 2018). Dazu gehört auch die Forschung zu digitalisierten Öffentlichkeiten, die mögliche Konsequenzen wie deren Zerfall (Jarren et al., 2000) und eine durch das Internet ausgelöste gesellschaftliche Fragmentierung untersucht (Mahrt, 2019; Webster \& Ksiazek, 2012; Zuiderveen Borgesius et al., 2016) und dabei u. a. Kommunikationsphänomene wie Echokammern auf vermeintliche Filterblasen in den virtuellen Räumen übertragen hat (Pariser, 2011; Sunstein, 2002).

Die vorhergehenden Ausführungen dienen an dieser Stelle lediglich der Illustration der Vielseitigkeit sozial- und kommunikationswissenschaftlicher Forschung zur Digitalisierung. Sie dokumentieren jedoch, dass unter der Annahme einer fortschreitenden Digitalisierung eine tiefgreifende Beeinflussung der sozialen Situation durch die Präsenz von Phänomenen der Digitalisierung in Gegenwart und Zukunft und diesbezüglicher sozialer Kommunikation erwartet wird, die ein Verständnis und Erklären dieser Veränderungen für das soziale Handeln in seinem jeweiligen Alltagskosmos dringend notwendig machen. Dabei bedeutet die Präsenz von Phänomenen der Digitalisierung, dass nicht zwingend materielle Artefakte der Digitalisierung in einer Situation präsent sein müssen, sondern lediglich, dass die Auswirkungen einzelner Phänomene, die auf Digitalisierung zurückzuführen sind, in der sozialen Situation $\mathrm{zu}$ beobachten und mithin $\mathrm{zu}$ analysieren sind.

\subsection{Die öffentliche Wahrnehmung der (Un-) Wahrscheinlichkeit des Siegeszuges der Digitalisierung vor dem Hintergrund der Konzeption der Wissensgesellschaft}

Dabei ist Digitalisierung kein Selbstläufer. Wem blühende Landschaften versprochen werden und wer im Nachhinein merkt, dass man ihm oder ihr potemkinsche Dörfer präsentiert, bei dem oder der sollten auch hier Zweifel kommen, wie zuverlässig gemachte Prognosen für die Zukunft tatsächlich sind, insbesondere mit Blick auf die gesellschaftlichen Auswirkungen von Großtechnologien. Dass etwaige Versprechungen und Erwartungen nicht eingelöst werden, lässt sich mit Blick auf die Entwicklung bspw. der Biotechnologie (Joppi et al., 2005) oder der Atomkraft (U. Beck, 1987; Hasegawa, 2012, 2014) beobachten. Es besteht die Einsicht, dass technologische Entwicklung ohne eine gleichzeitige Berücksichtigung der potentiellen Risiken für die menschliche Gesellschaft undenkbar 
ist (U. Beck, 1986). So wäre zumindest zu erwarten, dass eine Person, die regelmäßig beobachtet, dass Voraussagen zu gesellschaftlichen Entwicklungen nicht wie versprochen und erwartet eintreten, die Eintrittswahrscheinlichkeit $P$ (Siegeszugder Digitalisierung) mit kleiner als 1 belegt. Zumal an dieser Stelle noch vollkommen unreflektiert ist, wie konkret sich dieser Siegeszug in unterschiedlichen Sphären des öffentlichen und privaten Lebens manifestiert. Ohne hier schon zu viel vorwegzunehmen: Der vermeintliche Siegeszug - so es denn einer sein sollte - ist auch immer wieder gespickt mit vielen kleinen negativen Auswirkungen für die Menschen in ihren Rollen als Bürger*innen, Arbeitnehmer*innen und Konsument*innen, wie nachfolgend an etlichen Stellen diskutiert wird. Das letzte Wort der zukünftigen Entwicklung von Digitalisierung in der digitalen Gesellschaft ist noch nicht gesprochen und Erfolgsverkündungen sind zu früh. Nun zeigt der Blick auf die Forschung auch, dass selbst das NichtEintreten von Vorhersagen nicht automatisch zu erdrückenden Zweifeln an einer Idee führen muss, sondern häufig das Gegenteil bewirkt (Festinger et al., 1964). Mit anderen Worten: Auch wenn objektiv nicht von einem umfassenden Erfolg der Digitalisierung gesprochen werden kann, so sagt das noch lange nichts über den subjektiven Eindruck und dessen Bewertung aus.

Ohne jedoch hier schon auf eine mögliche Beantwortung der Fragen hinzuwirken, welche konkreten gesellschaftlichen Auswirkungen Digitalisierung zeitigt und ob und warum sie von der Bevölkerung als eine positive oder negative Entwicklung bewertet wird sowie welche Hoffnungen sich mit ihr für die Zukunft verbinden (siehe hierzu die Kapitel 4 und 5), soll zunächst folgende These aufgestellt werden: Digitalisierung ist mit Blick auf die mannigfaltige Verwendung des Begriffs im Alltag, in den Medien, in Wahlkämpfen und in der Wirtschaft eine derzeit wirkmächtige Idee. Sie nährt sich aus vielfach formulierten Erwartungen bezüglich der Konsequenzen bspw. der digitalen Schule, Verwaltung oder Gesundheitsversorgung, neuer Wirtschaftszweige und gänzlich veränderter Medien- und Informationsnutzung.

Der öffentliche Diskurs über die Digitalisierung und ihre spezifische Ausgestaltung, bspw. in Form des Phänomens Big Data, hat einen Einfluss darauf, was Menschen bei Fragen der Digitalisierung denken und wie sie sich zu diesen verhalten (Knorre et al., 2020). Analog zu theoretischen Überlegungen der Kultivierungsanalyse (Gerbner, 1973; Gerbner et al., 1980; Gerbner, 1998; Morgan, 2008) erfolgt hier eine in dieser Arbeit nachfolgend zu skizzierende Realitätsbeschreibung des Wesens und der Konsequenzen von Digitalisierung. Individuum und Gesellschaft evaluieren und lernen im Rahmen dieser öffentlichen kommunikativen Verhandlung laufend und langfristig, welchen vermeintlichen 
Entwicklungsstatus die digitale Gesellschaft hat und welchen mutmaßlichen Entwicklungsverlauf sie nimmt. Dabei formen sich in einem Prozess aufeinander aufbauender und miteinander verwobener Rezeptions- und Wirkungsereignisse insbesondere massenmedial geprägte Realitätskonzeptionen (Fahr \& Früh, 2011; Früh, 1991; Früh \& Schönbach, 1982; Gerbner, 1973; McQuail \& Windahl, 1993). Diese Realitätskonzeptionen sind also vor dem Hintergrund eines vielschichtigen öffentlichen Diskurses zu bewerten, aus dem sie sich speisen, in den sie jedoch auch wieder zurückwirken, wobei sich wechselseitige Transformationsprozesse einstellen.

In diesem Diskurs spielen - weit oberhalb des täglichen Klein-Kleins aktueller Geschehnisse - übergeordnete Gegenwartsdiagnosen eine zentrale und gewichtige Rolle für das Selbstverständnis einer Gesellschaft. Sie prägen als ausformulierte Erzählung einer gesamtgesellschaftlichen Selbstreflexion eine zeitgenössische Haltung, die sich im alltäglichen Handeln in den Sphären des öffentlichen und privaten Lebens niederschlägt. Die bereits angesprochene digitale Gesellschaft ist eine solche verhältnismäßig junge Gesellschaftsdiagnose. Sie erwächst jedoch einer weitaus allgemeineren Konzeption gesellschaftlicher Verfasstheit, die einen konkreten Bezug auf das epistemische Selbstverständnis des Menschen hat, in dessen Rahmen Digitallogik und Digitaltechnik eine maßgebliche und unverzichtbare Bedeutung für das Entstehen von Erkenntnis besitzen, jedoch auf den ersten Blick lediglich einen funktionalen Charakter innehaben (Nassehi, 2019). Gemeint ist die Zeitdiagnose der Wissensgesellschaft (Kübler, 2009; Stehr, 2012, 2017), auf die nachfolgend einzugehen ist (siehe Abschnitt 4.2). In ihrem Rahmen wird Wissen in einen Stand erhoben, der die faktische jedoch auch imaginierte besondere Bedeutung von Wissen für die Gesellschaft hervorhebt. Wissen ist dann zentrale Kategorie, doch gleichzeitig auch ein abstrahiertes und theoriegeladenes Konstrukt, über das gelernt und gewusst, diesbezüglich jedoch auch erwartet, gehofft und geglaubt werden kann (siehe Abschnitt 4.1). Es ergibt sich mithin abseits jeglicher tatsächlich manifestierter Konsequenz eine Erwartungshaltung, die auf die Wissensgesellschaft und das in ihr generierte Wissen gerichtet ist. Diese allgemeine Erwartungshaltung zielt dann auf die Idee der Wissensgesellschaft ab, die zwar vielfach und immer anders nuanciert diskutiert wird, zu deren Kernbestand jedoch meist alle Innovationen und Artefakte aus dem Bereich der IuK zählen (Gerbner et al., 1973; Kübler, 2009).

\section{Digitale Daten als Kernelement der digitalisierten Wissensgesellschaft}

Die vorliegende Arbeit nimmt dabei nun den Verwertungszusammenhang digitaler Daten in den Blick, die im Rahmen der fortschreitenden Digitalisierung bei der Verwendung eben jener IuK entstehen, und die gesellschaftliche Bedeutung dieser 
Daten sowie öffentliche Erwartungen hinsichtlich Erkenntnis- und Nutzengewinnen (Houben \& Prietl, 2018; Knorre et al., 2020; Kolany-Raiser et al., 2018). Denn aus der Digitalisierung, die in ihrem Wesen aus einer Unterscheidung in Nullen und Einsen besteht, ergibt sich eine konstante und gewichtige Konsequenz: Digitalisierung ist datengetrieben. Grund hierfür ist vor allem, dass die technische Möglichkeit der Feststellung und Übertragung realweltlicher Zustände in ein binäres digitales Format und dessen langfristige Speicherung Wissen über Zustände jenseits der Technik bereitstellt. Hieraus abgeleitete Erkenntnisse über die Welt können in vielfältigen sozialen Kontexten aufgegriffen, verwertet und genutzt werden und regen somit Anschlusshandlungen an. Daher sind digitale Daten, so eine weitere Prämisse der vorliegenden Arbeit, Ergebnis und gleichzeitig Treiber der Digitalisierung der Gesellschaft in einem rekursiven Prozess. Dieser dehnt sich - nicht nur, weil es technisch möglich ist, sondern weil es gesellschaftlich befürwortet wird - auf immer mehr Lebensbereiche aus und führt zu sozialen Konsequenzen für Individuum und Gesellschaft. Die digitalen Daten sowie vor allem das auf ihnen aufbauende Wissen erfahren hierbei unter Stichworten wie Big Data, Datafizierung oder das auf Daten basierende Machine Learning (ML) und hiermit verbundener Erwartungen an Künstliche Intelligenz (KI) derzeit eine hohe Aufmerksamkeit in öffentlichen Diskussionen der Sphären der Wissenschaft, der Wirtschaft und Politik sowie der Zivilgesellschaft (Finlay, 2017; J. Kaplan, 2016; Katz, 2017; Knorre et al., 2020; Mayer-Schönberger \& Cukier, 2013; O’Neil, 2017; Orwat \& Schankin, 2018).

Bevor jedoch auf die gesellschaftlichen Bedingungen und Erwartungen bezüglich der digitalen Datensammlung und -verwertung vor dem Hintergrund eines Erkenntnis- und Nutzengewinns eingegangen werden kann, ist zunächst Folgendes zu klären: Was genau hat es mit digitalen Daten auf sich und in welchem Zusammenhang stehen sie zum Wissen? Was bedeutet hierbei überhaupt Wissen und welche Bedeutung hat es für Gesellschaft und Digitalisierung? Es wird daher im nachfolgenden Kapitel 3 zunächst einmal abgegrenzt, was unter digitalen Daten verstanden wird und welche Voraussetzungen und Konsequenzen mit ihrer Entstehung, Sammlung und Auswertung in technischer wie in sozialer Hinsicht verbunden sind. Nach der Diskussion dieser vor allem sozio-technischen Voraussetzungen von digitalen Daten werden dann in Kapitel 4 das Wesen und die Bedeutung des Wissens in den Blick genommen, das auf Grundlage digitaler Daten gewonnen werden kann. Erst im Anschluss kann sich der Analyse der sozialen Bedeutung von Big Data und Wissen für die digitalisierte Gesellschaft vor dem Hintergrund aktueller gesellschaftlicher Entwicklungen gewidmet werden. 
Open Access Dieses Kapitel wird unter der Creative Commons Namensnennung 4.0 International Lizenz (http://creativecommons.org/licenses/by/4.0/deed.de) veröffentlicht, welche die Nutzung, Vervielfältigung, Bearbeitung, Verbreitung und Wiedergabe in jeglichem Medium und Format erlaubt, sofern Sie den/die ursprünglichen Autor(en) und die Quelle ordnungsgemäß nennen, einen Link zur Creative Commons Lizenz beifügen und angeben, ob Änderungen vorgenommen wurden.

Die in diesem Kapitel enthaltenen Bilder und sonstiges Drittmaterial unterliegen ebenfalls der genannten Creative Commons Lizenz, sofern sich aus der Abbildungslegende nichts anderes ergibt. Sofern das betreffende Material nicht unter der genannten Creative Commons Lizenz steht und die betreffende Handlung nicht nach gesetzlichen Vorschriften erlaubt ist, ist für die oben aufgeführten Weiterverwendungen des Materials die Einwilligung des jeweiligen Rechteinhabers einzuholen.

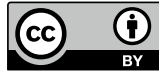

